\title{
AN ASYMMETRIC ST. PETERSBURG GAME WITH TRIMMING
}

\author{
BY ALLAN GUT AND ANDERS MARTIN-LÖF
}

\begin{abstract}
Let $S_{n}, n \geq 1$, be the successive sums of the payoffs in the classical St. Petersburg game. The celebrated Feller weak law states that $S_{n} /\left(n \log _{2} n\right) \stackrel{\mathbb{P}}{\rightarrow} 1$ as $n \rightarrow \infty$. In this paper we review some earlier results of ours and extend some of them as we consider an asymmetric St. Petersburg game, in which the distribution of the payoff $X$ is given by $\mathbb{P}\left(X=s r^{k-1}\right)=p q^{k-1}, k=1,2, \ldots$, where $p+q=1$ and $s, r>0$. Two main results are extensions of the Feller weak law and the convergence in distribution theorem of Martin-Löf (1985). Moreover, it is well known that almost-sure convergence fails, though Csörgó and Simons (1996) showed that almost-sure convergence holds for trimmed sums and also for sums trimmed by an arbitrary fixed number of maxima. In view of the discreteness of the distribution we focus on 'max-trimmed sums', that is, on the sums trimmed by the random number of observations that are equal to the largest one, and prove limit theorems for simply trimmed sums, for max-trimmed sums, as well as for the 'total maximum'. Analogues with respect to the random number of summands equal to the minimum are also obtained and, finally, for joint trimming.
\end{abstract}

Keywords: St. Petersburg game; sums of independent and identically distributed random variables; Feller's weak law of large numbers; convergence along subsequences; convergence in distribution; trimmed sum

2010 Mathematics Subject Classification: Primary 60F05; 60G50

Secondary 26A12; 60F15

\section{Introduction}

Th classical St. Petersburg game is defined as follows (see, e.g. [9]). Peter throws a fair coin repeatedly until heads turns up; if this happens at trial number $k$, he has to pay Paul $2^{k}$ ducats. What might the value of the game be to Paul? Since the random variable $X$ describing the payoff is governed by

$$
\mathbb{P}\left(X=2^{k}\right)=\frac{1}{2^{k}}, \quad k=1,2, \ldots,
$$

which has infinite expectation, this expected value gives no guidance as to what may be a 'fair' price for Paul to participate in the game.

One variation is to set the fee as a function of the number of games. This leads to the celebrated Feller solution [9], namely, that if $X, X_{1}, X_{2}, \ldots$ are independent and identically distributed (i.i.d.) random variables as above, and $S_{n}=\sum_{k=1}^{n} X_{k}, n \geq 1$, then

$$
\frac{S_{n}}{n \log _{2} n} \stackrel{\mathbb{P}}{\rightarrow} 1 \quad \text { as } n \rightarrow \infty,
$$

where, generally, $\log _{r}(\cdot)$ denotes the logarithm relative to the base $r>0$. For details, see [10, Chapter X] and [11, Chapter VII] or [14, Section 6.4.1]. More on the history of the game can be found in [18]. 
This paper is devoted to the generalization in which a biased coin, for which $\mathbb{P}$ (heads) $=p$, $0<p<1$, is tossed repeatedly until heads appears. If this happens at trial number $k$, you receive $s r^{k-1}$ euro, where $s, r>0$. This procedure induces the random variable

$$
\mathbb{P}\left(X=s r^{k-1}\right)=p q^{k-1}, \quad k=1,2, \ldots
$$

Our first result is an extension of Feller's weak law (1.1) to the setting (1.2) under the assumption that $r=1 / q$. If, in addition, $s=1 / p$, the result reduces to [13, Theorem 2.1(i)], where additional references can be found. The case in which $p=q=\frac{1}{2}$ (and $s=1 / p$ ) corresponds to the classical game.

As for convergence in distribution in the classical case, Martin-Löf [18] obtained convergence in distribution along the geometric subsequence $2^{n}$ to an infinitely divisible, semi-stable distribution. In Section 2 we extend his theorem to the general case. If $s=1 / p$ and $r=1 / q$ then some of our results reduce to those of [13], while if also $p=q=\frac{1}{2}$, we are in the setting of [18].

Next note that in the setting of Feller's law (1.1) almost-sure convergence fails because

$$
\liminf _{n \rightarrow \infty} \frac{S_{n}}{\log _{2} n}=1 \quad \text { and } \quad \limsup _{n \rightarrow \infty} \frac{S_{n}}{\log _{2} n}=+\infty
$$

(see [1, Example 4] and [5], respectively). However, Csörgő and Simons [8] showed that almost-sure convergence holds for trimmed sums, that is, for $S_{n}-\max _{1 \leq k \leq n} X_{k}$, as well as for sums trimmed by an arbitrary fixed number of maxima (for a recent paper on this, see [4]). Since our actual distribution is discrete, there may be ties. We therefore focus on 'max-trimmed sums', that is, on sums trimmed by the random number of observations that are equal to the largest one. In Section 3 we prove an analogue of Martin-Löf's distributional limit theorem [18] for such max-trimmed sums, as well as for simply trimmed sums and the 'total maxima', after which we interpret these findings in terms of sums of truncated Poisson random variables in Section 5.

The, albeit maybe less important, min-trimming amounts to subtracting the random number of minima from the sums. This is the topic of Section 6, after which in Section 7 we provide some remarks on joint trimming, which might be interpreted as a random version of intermediate trimming. These results extend those of Gut and Martin-Löf [15], [17], which are devoted to the classical case. A final section contains some remarks on almost-sure convergence.

\section{A weak law and a limit distribution}

Throughout this paper, let $X, X_{1}, X_{2}, \ldots$ be i.i.d. random variables with

$$
\mathbb{P}\left(X=s r^{k-1}\right)=p q^{k-1}, \quad k=1,2, \ldots,
$$

and set $S_{n}=\sum_{k=1}^{n} X_{k}$ and $M_{n}=\max _{1 \leq k \leq n} X_{k}, n \geq 1$. Since we are aiming at weak limits, we begin by noting that if $r<1 / q$ then $\mathbb{E}[X]<\infty$, so that the classical strong law holds, viz.

$$
\frac{S_{n}}{n} \stackrel{\text { a.s. }}{\longrightarrow} \frac{s p}{1-r q} \text { as } n \rightarrow \infty,
$$

where, for the value of the limit, we refer to (2.2) below with $\beta=1$.

In the following we therefore assume that $r q \geq 1$, and, thus, in particular, that $r>1$. 
Theorem 2.1. If $r=1 / q$ then

$$
\frac{S_{n}}{n \log _{r} n} \stackrel{\mathbb{P}}{\rightarrow} \text { sp } \quad \text { as } n \rightarrow \infty
$$

Remark 2.1. For $p=q=\frac{1}{2}$ and $s=2$, the theorem reduces to (1.1); for general $p$ with $s=1 / p$, it reduces to [13, Theorem 2.1(i)]. For the case $s=r=q^{-1}$, Adler and Rosalsky [2, Theorem 4] proved a weak law for the weighted sum $\sum_{k=1}^{n} k^{\gamma} X_{k}$, where $\gamma>-1$.

Our next theorem extends Martin-Löf's subsequence result for the classical game [18]. Note that $M, N$, and $t N$ below need not be integers; we leave it to the reader to replace such quantities with the respective integer parts and to make the necessary amendments.

Theorem 2.2. Let $N=r^{n}$ and $M=q^{-n}$.

(i) If $r=1 / q$ then, for $t>0$,

$$
\frac{S_{t N}-t s p N n}{N}=\frac{S_{t N}}{N}-t s p n \stackrel{\mathrm{D}}{\rightarrow} Z(t) \quad \text { as } n \rightarrow \infty,
$$

where $Z(t)$ is the Lévy process defined via the characteristic function $\varphi_{Z(t)}(u)=$ $\mathbb{E}[\exp \{\mathrm{i} u Z(t)\}]=\exp \{t h(u)\}$, where

$$
h(u)=\sum_{k=-\infty}^{0}\left(\exp \left\{\mathrm{i} u s r^{k}\right\}-1-\mathrm{i} u s r^{k}\right) p q^{k}+\sum_{k=1}^{\infty}\left(\exp \left\{\mathrm{i} u s r^{k}\right\}-1\right) p q^{k} .
$$

(ii) If $r>1 / q$ then, for $t>0$,

$$
\frac{S_{t M}}{N} \stackrel{\mathrm{D}}{\rightarrow} Z(t) \quad \text { as } n \rightarrow \infty
$$

where now $Z(t)$ is defined via the characteristic function $\varphi_{Z(t)}(u)=\exp \{\operatorname{th}(u)\}$ with

$$
h(u)=\sum_{k=-\infty}^{\infty}\left(\exp \left\{\mathrm{i} u s r^{k}\right\}-1\right) p q^{k}
$$

In complete analogy with [18] we infer that the limit law is infinitely divisible, that the corresponding Lévy measure has point masses $p q^{k}$ at the points $s r^{k}$ for $k \in \mathbb{Z}$, and that we are facing a compound Poisson distribution with (two-sided) geometric weights. In addition, by replacing $2^{m}$ by $q^{-m}=r^{m}$ in the proof of [18, Theorem 2], it follows immediately that $h(u)=$ $q^{m}\left(h\left(u q^{m}\right)+\mathrm{i} u s p m\right)$ for all $m \in \mathbb{Z}$, and, hence, that the limit distribution in Theorem 2.2(i) is semi-stable in the sense of Lévy, which illustrates the fact that we do not have a limit distribution for the full sequence.

Next we present some technicalities, after which we present the proofs of the theorems.

\subsection{Preliminaries}

The following well-known relation holds between logarithms with bases $r$ and $u$ for $y>0$ :

$$
\log _{r}(y)=\log _{u}(y) \cdot \log _{r}(u)
$$


Lemma 2.1. For $X$ as defined in Theorem 2.1,

$$
\mathbb{E}\left[X^{\beta}\right]= \begin{cases}\frac{s^{\beta} p}{1-r^{\beta} q} & \text { for } r<q^{-1 / \beta} \\ +\infty & \text { for } r \geq q^{-1 / \beta}\end{cases}
$$

Moreover, as $x \rightarrow \infty$,

$$
\begin{gathered}
\mathbb{P}(X>x)=q^{\left[\log _{r}(x / s)\right]+1} \geq q^{\log _{r}(x / s)+1} \geq \frac{s}{r x}, \\
\mathbb{E}[X \mathbf{1}\{X \leq x\}] \sim \operatorname{sp} \log _{r}\left(\frac{x}{s}\right) \text { when } r=\frac{1}{q} .
\end{gathered}
$$

Proof. Relation (2.2) follows via the fact that $\mathbb{E}\left[X^{\beta}\right]=\sum_{k=1}^{\infty}\left(s r^{k-1}\right)^{\beta} p q^{k-1}$, and the tail estimate is equivalent to [7, Equation (1)]. The final inequality exploits the fact that $r q \geq 1$.

Relation (2.4) for the truncated first moment for large $x$ follows directly from

$$
\mathbb{E}[X \mathbf{1}\{X \leq x\}]=\sum_{\left\{k: s r^{k-1} \leq x\right\}} s r^{k-1} p q^{k-1}=s p \sum_{1 \leq k \leq \log _{r}(x / s)+1} 1=s p\left[\log _{r}\left(\frac{x}{s}\right)+1\right] .
$$

\subsection{Proof of Theorem 2.1}

Recall that $r=1 / q$. Observe first that the function $x \log _{1 / q} x \in \mathcal{R V}(1)$ (that is, regularly varying with exponent 1$)$.

Now, $n \mathbb{P}\left(X>n \log _{r} n\right)=n q^{\left[\log _{r}\left((n / s) \log _{r} n\right)\right]+1} \sim n /\left((n / s) \log _{r} n\right)=s / \log _{r} n \rightarrow 0$ and $\mathbb{E}\left[X \mathbf{1}\left\{X \leq n \log _{r} n\right\}\right] \sim s p \log _{r}\left((n / s) \log _{r} n\right)$ as $n \rightarrow \infty$ by (2.3) and (2.4), respectively, so that

$$
\frac{n \mathbb{E}\left[X \mathbf{1}\left\{X \leq n \log _{r} n\right\}\right]}{n \log _{r} n} \rightarrow 0,
$$

after which the conclusion is an immediate consequence of the extension of Feller's weak law of large numbers given in [12, Theorem 1.3]; cf. also [14, Theorem 6.4.2].

\subsection{Proof of Theorem 2.2}

Theorem 2.2(i) is proved via a straightforward modification of the corresponding proof in [18].

Since $\mathbb{P}\left(X=s r^{k-1}\right)=p q^{k-1}, \varphi_{X}(u)=\mathbb{E}\left[\mathrm{e}^{\mathrm{i} u X}\right]=\sum_{k=1}^{\infty} \mathrm{e}^{\mathrm{i} u s r^{k-1}} p q^{k-1}$, from which it follows that

$$
\begin{aligned}
\varphi_{S_{t N} / N-t s p n}(u) & =\mathrm{e}^{-\mathrm{i} u t s p n}\left(\sum_{k=1}^{\infty} \mathrm{e}^{\mathrm{i} u s r^{k} / N} p q^{k}\right)^{t N} \\
& =\mathrm{e}^{-\mathrm{i} u t s p n}\left(\sum_{k=1}^{\infty} \mathrm{e}^{\mathrm{i} u s r^{k-n}} p q^{k}\right)^{t N} \\
& =\mathrm{e}^{-\mathrm{i} u t s p n}\left(1+\sum_{k=1}^{\infty}\left(\mathrm{e}^{\mathrm{i} u s r^{k-n}}-1\right) p q^{k}\right)^{t N} \\
& =\mathrm{e}^{-\mathrm{i} u t s p n}\left(1+q^{n} \sum_{k=-n+1}^{\infty}\left(\mathrm{e}^{\mathrm{i} u s r^{k}}-1\right) p q^{k}\right)^{t N} \\
& =\mathrm{e}^{-\mathrm{i} u t s p n}\left(1+\frac{1}{N} \sum_{k=-n+1}^{\infty}\left(\mathrm{e}^{\mathrm{i} u s r^{k}}-1\right) p q^{k}\right)^{t N}
\end{aligned}
$$




$$
\begin{gathered}
=\mathrm{e}^{-\mathrm{i} u t s p n}\left(1+\frac{1}{N} \sum_{k=-n+1}^{0}\left(\mathrm{e}^{\mathrm{i} u s r^{k}}-1-i u s r^{k}\right) p q^{k}\right. \\
\left.+\mathrm{i} u s p \frac{n}{N}+\frac{1}{N} \sum_{k=1}^{\infty}\left(\mathrm{e}^{\mathrm{i} u s r^{k}}-1\right) p q^{k}\right)^{t N} \\
=\mathrm{e}^{-\mathrm{i} u t s p n}\left(1+\frac{1}{N}\left\{\sum_{k=-n+1}^{0}\left(\mathrm{e}^{\mathrm{i} u s r^{k}}-1-\mathrm{i} u s r^{k}\right) p q^{k}\right.\right. \\
\left.\left.+\mathrm{i} u s p n+\sum_{k=1}^{\infty}\left(\mathrm{e}^{\mathrm{i} u s r^{k}}-1\right) p q^{k}\right\}\right)^{t N},
\end{gathered}
$$

which converges to $\mathrm{e}^{t h(u)}$ as $n \rightarrow \infty$. The proof of (ii) is similar with obvious modifications.

\section{Max-trimming}

For distributions with infinite variance that belong to some domain of attraction, a sum is large because one of the summands is large. In fact, recalling (1.3), the process has rather wild sample behaviour. It is then frequently natural to consider sums minus the maximum and hope for some 'nicer' behaviour. This has generated the theory of trimmed sums. In the present context Csörgó and Simons [8] showed that a strong law holds for the trimmed sums, namely,

$$
\frac{S_{n}-\max _{1 \leq k \leq n} X_{k}}{n \log _{2} n} \stackrel{\text { a.s. }}{\longrightarrow} 1 \text { as } n \rightarrow \infty ;
$$

indeed, they proved more, namely that almost-sure convergence holds for the sums trimmed by any finite number of summands.

Rather than focussing on a fixed number of large observations, our interest lies in what we call 'max-trimmed' sums, that is, in the successive partial sums minus all maximal observations. This means that we trim the sums by the random number of summands equal to the largest one-this last we call the 'total maximum'. In Section 4 we prove analogues for the maxtrimmed sums, the trimmed sums, and the total maximum. Section 5 contains some remarks on identifications and interpretations of our results. For $p=q=\frac{1}{2}$ and $s=r=2$, our results reduce to those of [17]. Also, some of our results exist essentially as special cases of more general results (cf. [3], [6], and [7], where further references can be found). An important point is that here we provide more elementary and transparent proofs.

\subsection{Notation}

Trimming involves extreme observations, so we recall standard notation for order statistics,

$$
X_{1: n} \leq X_{2: n} \leq \cdots \leq X_{n: n},
$$

and note that equality between the order variables has positive probability because we have a discrete distribution. In particular, and this is a main point here, there is a random number of payoffs equal to the largest one. We therefore define

$$
\begin{gathered}
M_{n}=\max \left\{X_{1}, X_{2}, \ldots, X_{n}\right\} \quad\left(=X_{n: n}\right), \\
\tau_{n}^{(+)}=\#\left\{\text { obs }=M_{n}\right\}=\#\left\{k \leq n: X_{k}=M_{n}\right\}, \\
M_{n}^{*}=\sum_{k=n-\tau_{n}^{(+)}+1}^{n} X_{k: n}=\tau_{n}^{(+)} M_{n} .
\end{gathered}
$$


The classical trimmed sum equals $S_{n}-M_{n}$, that is, the sum minus the largest observation. Somewhat more generally, we may trim by subtracting more than one of the extremes. Our interest here concerns $S_{n}-M_{n}$ and $S_{n}-M_{n}^{*}$.

Finally, for any random variable $Y, \varphi_{Y}(u)=\mathbb{E}\left[\mathrm{e}^{\mathrm{i} u Y}\right]$ denotes its characteristic function and $g_{Y}(u)=\mathbb{E}\left[u^{Y}\right]$ its generating function.

\subsection{Some facts about $M_{n}$}

For later use, note also that, for any positive integer $m$,

$$
\begin{aligned}
& \mathbb{P}\left(X>s r^{m-1}\right)=q^{m}, \\
& \mathbb{P}\left(M_{n} \leq x\right)=\left(\sum_{\left\{k: s r^{k-1} \leq x\right\}} p q^{k-1}\right)^{n}=\left(1-q^{-\left[\log _{r} x / s\right]+1}\right)^{n}, \\
& \mathbb{P}\left(M_{n} \leq s r^{m-1}\right)=\left(1-q^{m}\right)^{n} .
\end{aligned}
$$

\subsection{The joint distribution of $M_{n}$ and $\tau_{n}^{(+)}$}

The event $\left\{\tau_{n}^{(+)}=k, M_{n}=s r^{m-1}\right\}$ occurs precisely when $k$ of the summands are equal to $s r^{m-1}$ and all others are at most equal to $s r^{m-2}$. This shows that, for $k=1,2, \ldots, n$ and $m \geq 1$,

$$
\mathbb{P}\left(\tau_{n}^{(+)}=k, M_{n}=s r^{m-1}\right)=\left(\begin{array}{l}
n \\
k
\end{array}\right)\left(p q^{m-1}\right)^{k}\left(1-q^{m-1}\right)^{n-k}
$$

Recall that $N=r^{n}=q^{-n}$ throughout, and that, as $n \rightarrow \infty,\left(\begin{array}{c}t N \\ k\end{array}\right) N^{-k} \rightarrow t^{k} / k !$. Then we conclude that, for $1 \leq k \leq t N$ and $m+n \geq 1$, as $n \rightarrow \infty$,

$$
\begin{aligned}
\mathbb{P}\left(\tau_{t N}^{(+)}=k, \frac{M_{t N}}{N}=s r^{m-1}\right) & =\left(\begin{array}{c}
t N \\
k
\end{array}\right)\left(\frac{p q^{m-1}}{N}\right)^{k}\left(1-\frac{q^{m-1}}{N}\right)^{t N-k} \\
& \rightarrow \frac{\left(t p q^{m-1}\right)^{k}}{k !} \mathrm{e}^{-t q^{m-1}} .
\end{aligned}
$$

\subsection{Marginal limits}

Let $M(t), t>0$, denote the random variable with the limiting distribution of $M_{t N} / N$ as $N \rightarrow \infty$ (equivalently, $n \rightarrow \infty$ ). Summing over $k$ in (3.5) and noting that $q^{m-1}=p q^{m-1}+q^{m}$, we obtain the following result.

Theorem 3.1. It holds that $M_{t N} / N \stackrel{\mathrm{D}}{\rightarrow} M(t)$ as $n \rightarrow \infty$, where, for $m=1,2, \ldots$,

$$
\mathbb{P}\left(M(t) \leq s r^{m-1}\right)=\mathrm{e}^{-t q^{m}} ; \text { hence, } \mathbb{P}\left(M(t)=s r^{m-1}\right)=\mathrm{e}^{-t q^{m}}\left(1-\mathrm{e}^{-t p q^{m-1}}\right) .
$$

By summing over $m$ in (3.4), we obtain the marginal limit for $\tau_{t N}^{(+)}$as follows.

Theorem 3.2. It holds that $\tau_{t N}^{(+)} \stackrel{\mathrm{D}}{\rightarrow} T(t)$ as $n \rightarrow \infty$, where

$$
\mathbb{P}(T(t)=k)=\frac{p^{k}}{k !} \sum_{m=-\infty}^{\infty}\left(t q^{m}\right)^{k} \mathrm{e}^{-t q^{m}} ; \text { hence, } \quad g_{T(t)}(u)=\sum_{m=-\infty}^{\infty}\left(\mathrm{e}^{u p t q^{m}}-1\right) \mathrm{e}^{-t q^{m}}
$$




\subsection{The generating function of $\tau_{n}^{(+)}$}

Proposition 3.1. The probability generating function of $\tau_{n}^{(+)}$is given by

$$
g_{\tau_{n}^{(+)}}(u)=\sum_{m=1}^{\infty}\left[\left(1-q^{m-1}(1-p u)\right)^{n}-\left(1-q^{m-1}\right)^{n}\right] .
$$

Proof. Exploiting (3.4) we obtain

$$
\begin{aligned}
g_{\tau_{n}^{(+)}}(u) & =\sum_{k=1}^{n} u^{k} \mathbb{P}\left(\tau_{n}^{(+)}=k\right) \\
& =\sum_{k=1}^{n} u^{k} \sum_{m=1}^{\infty} \mathbb{P}\left(\tau_{n}^{(+)}=k, M_{n}=s r^{m-1}\right) \\
& =\sum_{m=1}^{\infty} \sum_{k=1}^{n} u^{k}\left(\begin{array}{l}
n \\
k
\end{array}\right)\left(p q^{m-1}\right)^{k}\left(1-q^{m-1}\right)^{n-k} \\
& =\sum_{m=1}^{\infty}\left[\left(1-q^{m-1}(1-p u)\right)^{n}-\left(1-q^{m-1}\right)^{n}\right] .
\end{aligned}
$$

By differentiating the generating function, or else by exploiting (3.4) as above, it is now easy to determine, for example, the individual probabilities and moments of $\tau_{n}^{(+)}$.

\section{Asymptotics}

In this section we extend our results from [17] by proving analogues of Martin-Löf's limit theorem [18] for max-trimmed sums, trimmed sums, and the total maximum.

\subsection{The max-trimmed sum $S_{t N}-M_{t N}^{*}$}

Theorem 4.1. Let $N=r^{n}=q^{-n}$ as before, and define

$$
h_{m}(u)=\sum_{j=-\infty}^{m}\left(\mathrm{e}^{\mathrm{i} u s r^{j-1}}-1-\mathrm{i} u s r^{j-1}\right) p q^{j-1}+\mathrm{i} \text { upsm. }
$$

Then, for $t>0$,

$$
\frac{S_{t N}-M_{t N}^{*}}{N}-p s t n \stackrel{\mathrm{D}}{\rightarrow} Z^{*}(t) \quad \text { as } n \rightarrow \infty,
$$

where $Z^{*}(t)$ has the characteristic function

$$
\mathbb{E}\left[\mathrm{e}^{\mathrm{i} u Z^{*}(t)}\right]=\sum_{m=-\infty}^{\infty} \mathrm{e}^{t h_{m-1}(u)} \mathrm{e}^{-t q^{m}}\left(1-\mathrm{e}^{-t p q^{m-1}}\right) .
$$

Proof. Let $m \geq 1$, define $\psi_{m}(u)=\sum_{k=1}^{m} \mathrm{e}^{\mathrm{i} u s r^{k-1}} p q^{k-1}$, and, for $k \geq 1$, write $X_{k}^{(m)}$ for $X_{k}$ conditional on $X_{k} \leq s r^{m-1}$. Then $\varphi_{X^{(m)}}(u)=\mathbb{E}\left[\mathrm{e}^{\mathrm{i} u X^{(m)}}\right]=\psi_{m}(u) /\left(1-q^{m}\right)$; hence,

$$
\mathbb{E}\left[\exp \left\{\mathrm{i} u \sum_{j=1}^{n} X_{j}^{(m)}\right\}\right]=\left(\frac{\psi_{m}(u)}{1-q^{m}}\right)^{n},
$$

so that

$$
\mathbb{E}\left[\exp \left\{\frac{\mathrm{i} u}{N} \sum_{j=1}^{t N-k} X_{j}^{(m+n-1)}\right\}\right]=\left(\frac{\psi_{m+n-1}(u / N)}{1-q^{m+n-1}}\right)^{t N-k} .
$$


We can express $\psi$ as

$$
\begin{aligned}
\psi_{m+n-1}\left(\frac{u}{N}\right) \\
=\sum_{j=1}^{m+n-1} \mathrm{e}^{\mathrm{i}(u / N) s r^{j-1}} p q^{j-1} \\
=\sum_{j=-n+1}^{m-1} \mathrm{e}^{\mathrm{i} u s r^{j-1}} p q^{n+j-1} \\
=\sum_{j=-n+1}^{m-1}\left(\left(\mathrm{e}^{\mathrm{i} u s r^{j-1}}-1-\mathrm{i} u s r^{j-1}\right) p q^{n+j-1}+\left(1+\mathrm{i} u s r^{j-1}\right) p q^{n+j-1}\right) \\
=\frac{1}{N} \sum_{j=-n+1}^{m-1}\left(\mathrm{e}^{\mathrm{i} u s r^{j-1}}-1-\mathrm{i} u s r^{j-1}\right) p q^{j-1}+\left(1-q^{n+m-1}\right)+\frac{\mathrm{i} u p s(m+n-1)}{N} \\
=1-\frac{q^{m-1}}{N}+\frac{\mathrm{i} u p s(m+n-1)}{N}+\frac{1}{N} \sum_{j=-n+1}^{m-1}\left(\mathrm{e}^{\mathrm{i} u s r^{j-1}}-1-\mathrm{i} u s r^{j-1}\right) p q^{j-1},
\end{aligned}
$$

so that, following the path of [17] and [18] cited above,

$$
\begin{aligned}
\mathbb{E}\left[\exp \left\{\mathrm{i} u\left(\frac{S_{t N}-M_{t N}^{*}}{N}-p s t n\right)\right\}, \tau_{t N}^{(+)}=k, \frac{M_{t N}}{N}=s r^{m-1}\right] \\
=\mathrm{e}^{-\mathrm{i} u p s t n} \mathbb{E}\left[\exp \left\{\frac{\mathrm{i} u}{N} \sum_{j=1}^{t N-k} X_{j}^{(m+n-1)}\right\}\right] \mathbb{P}\left(\tau_{t N}^{(+)}=k, M_{t N}=s r^{m+n-1}\right) \\
=\mathrm{e}^{-\mathrm{i} u p s t n}\left(\frac{\psi_{m+n-1}(u / N)}{1-q^{m+n-1}}\right)^{t N-k}\left(\begin{array}{c}
t N \\
k
\end{array}\right)\left(p q^{m+n-1}\right)^{k}\left(1-q^{m+n-1}\right)^{t N-k} \\
=\mathrm{e}^{-\mathrm{i} u p s t n}\left(1-\frac{q^{m-1}}{N}+\frac{\mathrm{i} u p s(m+n-1)}{N}\right. \\
\left.\quad+\frac{1}{N} \sum_{j=-n+1}^{m-1}\left(\mathrm{e}^{\mathrm{i} u s r^{j-1}}-1-\mathrm{i} u s r^{j-1}\right) p q^{j-1}\right)^{t N-k} \times\left(\begin{array}{c}
t N \\
k
\end{array}\right)\left(\frac{p q^{m-1}}{N}\right)^{k} .
\end{aligned}
$$

With $h_{m}(u)$ as defined in (4.1), standard estimates tell us that, as $n \rightarrow \infty$, the expression in (4.2) preceding ' $x$ ' converges to

$$
\mathrm{e}^{t h_{m-1}(u)-t q^{m-1}} \frac{\left(t p q^{m-1}\right)^{k}}{k !}=\mathrm{e}^{t h_{m-1}(u)} \mathrm{e}^{-t q^{m-1}} \frac{\left(t p q^{m-1}\right)^{k}}{k !},
$$

a quantity that, via an appeal to [14, Lemma A.1.2], is bounded. Therefore, since

$$
\left(\begin{array}{c}
t N \\
k
\end{array}\right)\left(\frac{p q^{m-1}}{N}\right)^{k} \leq \frac{\left(t p q^{m-1}\right)^{k}}{k !}
$$


we conclude that

$$
\begin{aligned}
\mathbb{E}[\exp & \left.\left\{\mathrm{i} u\left(\frac{S_{t N}-M_{t N}^{*}}{N}-p s t n\right)\right\}, \frac{M_{t N}}{N}=s r^{m-1}\right] \\
= & \sum_{k=1}^{t N} \mathbb{E}\left[\exp \left\{\mathrm{i} u\left(\frac{S_{t N}-M_{t N}^{*}}{N}-p s t n\right)\right\}, \tau_{t N}^{(+)}=k, \frac{M_{t N}}{N}=s r^{m-1}\right] \\
& \rightarrow \sum_{k=1}^{\infty} \mathrm{e}^{t h_{m-1}(u)} \mathrm{e}^{-t q^{m-1}} \frac{\left(t p q^{m-1}\right)^{k}}{k !} \quad(\text { as } N \rightarrow \infty), \\
= & \mathrm{e}^{t h_{m-1}(u)} \mathrm{e}^{-t q^{m-1}}\left(\mathrm{e}^{t p q^{m-1}}-1\right) \\
= & \mathrm{e}^{t h_{m-1}(u)} \mathrm{e}^{-t q^{m}}\left(1-\mathrm{e}^{-t p q^{m-1}}\right) .
\end{aligned}
$$

In order to complete the proof, we first note that

$$
\begin{array}{rl}
\sum_{m \geq-n+1} & \mathbb{E}\left[\exp \left\{\mathrm{i} u\left(\frac{S_{t N}-M_{t N}^{*}}{N}-p s t n\right)\right\}, \frac{M_{t N}}{N}=s r^{m-1}\right] \\
= & \sum_{m \geq-n+1} \mathbb{E}\left[\exp \left\{\mathrm{i} u\left(\frac{S_{t N}-M_{t N}^{*}}{N}-p s t n\right)\right\} \mid \frac{M_{t N}}{N}=s r^{m-1}\right] \mathbb{P}\left(\frac{M_{t N}}{N}=s r^{m-1}\right) \\
= & \sum_{m \geq-n+1} \mathbb{E}\left[\exp \left\{\mathrm{i} u\left(\frac{S_{t N}-M_{t N}^{*}}{N}-p s t n\right)\right\} \mid \frac{M_{t N}}{N}=s r^{m-1}\right] \mathrm{e}^{-t q^{m}}\left(1-\mathrm{e}^{-t p q^{m-1}}\right) \\
& +\sum_{m \geq-n+1} \mathbb{E}\left[\exp \left\{\mathrm{i} u\left(\frac{S_{t N}-M_{t N}^{*}}{N}-p s t n\right)\right\} \mid \frac{M_{t N}}{N}=s r^{m-1}\right] \\
\quad \times\left[\mathbb{P}\left(\frac{M_{t N}}{N}=s r^{m-1}\right)-\mathrm{e}^{-t q^{m}}\left(1-\mathrm{e}^{-t p q^{m-1}}\right)\right] & \\
:= & I+I I . \quad
\end{array}
$$

Furthermore,

$$
\begin{aligned}
& \left|\mathbb{E}\left[\exp \left\{\mathrm{i} u\left(\frac{S_{t N}-M_{t N}^{*}}{N}-p s t n\right)\right\} \mid \frac{M_{t N}}{N}=s r^{m-1}\right]\right| \\
& \quad \leq \mathbb{E}\left[\left|\exp \left\{\mathrm{i} u\left(\frac{S_{t N}-M_{t N}^{*}}{N}-p s t n\right)\right\}\right| \mid \frac{M_{t N}}{N}=s r^{m-1}\right] \\
& \quad=1
\end{aligned}
$$

so, with $d(\cdot, \cdot)$ denoting the variational distance,

$$
|I I| \leq 2 d\left(\frac{M_{t N}}{N}, M(t)\right) .
$$

This, finally, tells us that, as $n \rightarrow \infty$,

$$
I \rightarrow \sum_{m=-\infty}^{\infty} \mathrm{e}^{t h_{m-1}(u)} \mathrm{e}^{-t q^{m}}\left(1-\mathrm{e}^{-t p q^{m-1}}\right)
$$

by dominated convergence, and $I I \rightarrow 0$ by the discrete version of Scheffé's lemma (see [14, Theorem 5.6.4]). 


\subsection{The trimmed sum $S_{t N}-M_{t N}$}

Minor modifications of the arguments from the previous section, together with a glance at [17], allow us to establish analogous asymptotics for the trimmed sums: we start from the fact that the difference between a max-trimmed sum and the corresponding trimmed sum consists of the $\tau_{n}^{(+)}-1$ maximal observations which are subtracted in the former sum but not in the latter, namely,

$$
\left(S_{n}-M_{n}^{*}\right)-\left(S_{n}-M_{n}\right)=M_{n}-M_{n}^{*}=M_{n}-\tau_{n}^{(+)} M_{n}=-\left(\tau_{n}^{(+)}-1\right) M_{n} .
$$

Theorem 4.2. Let $N=r^{n}=q^{-n}$, and define $h_{m}(u)$ as in (4.1). Then, for $t>0$ and $n \rightarrow \infty$,

$$
\frac{S_{t N}-M_{t N}}{N}-p s t n \stackrel{\mathrm{D}}{\rightarrow} Z_{*}(t),
$$

where $Z_{*}(t)$ has the characteristic function

$$
\mathbb{E}\left[\mathrm{e}^{\mathrm{i} u Z_{*}(t)}\right]=\sum_{m=-\infty}^{\infty} \mathrm{e}^{-\mathrm{i} u s r^{m-1}+t h_{m-1}(u)-t q^{m-1}}\left(\mathrm{e}^{t p q^{m-1} \mathrm{e}^{\mathrm{i} u s r^{m-1}}}-1\right) .
$$

\subsection{The total maximum $M_{t N}^{*}$}

Finally, we provide the limiting characteristic function for the total maximum, $M_{t N}^{*}$. To this end, we again modify the corresponding proof in [17].

Theorem 4.3. Let $N=r^{n}=q^{n}$. Then, as $n \rightarrow \infty$,

$$
\mathbb{E}\left[\exp \left\{\mathrm{i} u \frac{M_{t N}^{*}}{N}\right\}\right] \rightarrow \sum_{m=-\infty}^{\infty} \mathrm{e}^{-t q^{m-1}}\left(\mathrm{e}^{t p q^{m-1} \mathrm{e}^{\mathrm{i} u s r^{m-1}}}-1\right) .
$$

Moreover, the mean of the limit distribution is infinite, in the sense that

$$
\mathbb{E}\left[\frac{M_{t N}^{*}}{N}\right] \rightarrow \operatorname{tsp} \sum_{m=-\infty}^{\infty} \mathrm{e}^{-t q^{m}}=+\infty
$$

\section{Interpretations and facts about the limiting processes}

In this section we interpret our limit theorems for max-trimmed sums, trimmed sums and max-trimming itself in terms of sums of Poisson random variables (RVs) and truncated Poisson RVs. Let Poi $(\lambda)$ denote a Poisson RV with mean $\lambda$; its characteristic function is $\varphi_{\operatorname{Poi}(\lambda)}(u)=$ $\exp \left\{\lambda\left(\mathrm{e}^{\mathrm{i} u}-1\right)\right\}$. For the Poisson process representation, see also [6] and [18]. As well as $h_{m}(u)$, defined in (4.1), recall $h(u)$ from Theorem 2.2(i), namely,

$$
h(u)=\sum_{j=-\infty}^{0}\left(\mathrm{e}^{\mathrm{i} u s r^{j-1}}-1-\mathrm{i} u s r^{j-1}\right) p q^{j-1}+\sum_{j=1}^{\infty}\left(\mathrm{e}^{\mathrm{i} u s r^{j-1}}-1\right) p q^{j-1} .
$$

By comparing $\mathrm{e}^{t h(u)}$ with $\varphi_{\operatorname{Poi}(\cdot)}$, we conclude that $\mathrm{e}^{t h(u)}$ is the characteristic function of

$$
Z(t)=\sum_{j=-\infty}^{0} s r^{j-1}\left(Z_{j}(t)-t p q^{j-1}\right)+\sum_{j=1}^{\infty} s r^{j-1} Z_{j}(t),
$$

where the $\left\{Z_{j}(t),-\infty<j<\infty\right\}$ are independent $\operatorname{Poi}\left(t p q^{j-1}\right)$-distributed random processes, 
and where $\mathrm{e}^{t h_{m}(u)}$ is the characteristic function of

$$
S_{m}(t)=\sum_{j=-\infty}^{m} s r^{j-1}\left(Z_{j}(t)-t p q^{j-1}\right)+p s t m .
$$

In terms of $\left\{Z_{j}\right\}$ this means that

$$
\begin{aligned}
\mathbb{P}\left(M(t)=s r^{m-1}\right) & =\mathbb{P}\left(Z_{m}(t)>0, Z_{j}(t)=0, j>m\right) \\
& =\left(1-\mathrm{e}^{-t p q^{m-1}}\right) \prod_{j=m+1}^{\infty} \mathrm{e}^{-t p q^{j-1}} \\
& =\left(1-\mathrm{e}^{-t p q^{m-1}}\right) \mathrm{e}^{-t q^{m}},
\end{aligned}
$$

in agreement with Theorem 3.1. Moreover, $S_{m-1}(t)$ is independent of $Z_{j}$ for all $j \geq m$.

\subsection{The max-trimmed sum}

Recalling from (3.3) that $M_{t N}^{*}=\tau_{t N}^{(+)} M_{t N}$, observe that, by exploiting Theorem 3.1, the limit in Theorem 4.1 can be rewritten as

$$
\frac{S_{t N}-M_{t N}^{*}}{N}-p s t n \stackrel{\mathrm{D}}{\rightarrow} Z^{*}(t) \quad \text { with } \quad \varphi_{Z^{*}(t)}(u)=\sum_{m=-\infty}^{\infty} \mathrm{e}^{t h_{m-1}(u)} \mathbb{P}\left(M(t)=s r^{m-1}\right) .
$$

\subsection{The trimmed sum}

Starting from (4.4) shows that, as $n \rightarrow \infty$,

$$
\begin{aligned}
\frac{S_{t N}-M_{t N}}{N}-p s t n & =\frac{S_{t N}-M_{t N}^{*}}{N}-p s t n+\left(\tau_{t N}^{(+)}-1\right) \frac{M_{t N}}{N} \\
& \stackrel{\mathrm{D}}{\rightarrow} Z^{*}(t)+(T(t)-1) M(t) \\
& \equiv Z_{*}(t) .
\end{aligned}
$$

Combining (5.1) and (3.5) then tells us that

$$
\begin{aligned}
\varphi_{Z_{*}(t)}(u) & =\sum_{m=-\infty}^{\infty} \sum_{k=1}^{\infty} \mathrm{e}^{\mathrm{i} u Z^{*}(t)} \mathrm{e}^{\mathrm{i} u(k-1) s r^{m-1}} \mathbb{P}\left(T(t)=k, M(t)=s r^{m-1}\right) \\
& =\sum_{m=-\infty}^{\infty} \sum_{k=1}^{\infty} \mathrm{e}^{t h_{m-1}(u)} \mathrm{e}^{\mathrm{i} u(k-1) s r^{m-1}} \frac{\left(t p q^{m-1}\right)^{k}}{k !} \mathrm{e}^{-t q^{m-1}} \\
& =\sum_{m=-\infty}^{\infty} \mathrm{e}^{-\mathrm{i} u s r^{m-1}+t h_{m-1}(u)-t q^{m-1}}\left(\mathrm{e}^{t p q^{m-1} \mathrm{e}^{\mathrm{i} u s r^{m-1}}}-1\right)
\end{aligned}
$$

\subsection{The total maximum}

As $n \rightarrow \infty$,

$$
\frac{M_{t N}^{*}}{N}=\tau_{t N}^{(+)} \frac{M_{t N}}{N} \stackrel{\mathrm{D}}{\rightarrow} T(t) M(t)=: M^{*}(t),
$$

for which the characteristic function is

$$
\varphi_{M^{*}(t)}(u)=\sum_{m=-\infty}^{\infty} \sum_{k=1}^{\infty} \mathrm{e}^{\mathrm{i} u k s r^{m-1}} \frac{\left(t p q^{m-1}\right)^{k}}{k !} \mathrm{e}^{-t q^{m-1}}=\sum_{m=-\infty}^{\infty} \mathrm{e}^{-t q^{m-1}}\left(\mathrm{e}^{t p q^{m-1} \mathrm{e}^{\mathrm{i} u s r^{m-1}}}-1\right) .
$$




\section{Min-trimming}

We now consider trimming with respect to the random number of summands equal to the minimum, $m_{n}$. In the classical case our findings reduce to those of [15]. In addition to the notation introduced in Section 3.1, notably (3.1)-(3.3), we define

$$
\begin{gathered}
m_{n}=\min \left\{X_{1}, X_{2}, \ldots, X_{n}\right\}, \\
\tau_{n}^{(-)}=\#\left\{\text { obs }=m_{n}\right\}=\#\left\{k \leq n: X_{k}=m_{n}\right\}, \\
m_{n}^{*}=\sum_{k=1}^{\tau_{n}^{(-)}} X_{k: n}=\tau_{n}^{(-)} m_{n} .
\end{gathered}
$$

\subsection{Some facts about $m_{n}$ and $\tau_{n}^{(-)}$}

For any positive integer $m$,

$\mathbb{P}\left(X_{1}>s r^{m-1}\right)=q^{m}, \quad \mathbb{P}\left(m_{n}>s r^{m-1}\right)=q^{m n}, \quad \mathbb{P}\left(m_{n}=s r^{m-1}\right)=q^{(m-1) n}\left(1-q^{n}\right)$.

Now, since $\sum_{n=1}^{\infty} \mathbb{P}\left(X_{n}=s\right)=\infty$, it follows from the second Borel-Cantelli lemma that $\mathbb{P}\left(X_{n}=s\right.$ i.o. $)=1$, which in turn shows in particular that

$$
m_{n} \stackrel{\text { a.s. }}{\longrightarrow} s \text { as } n \rightarrow \infty \text {; }
$$

indeed, $m_{n}=s$ for all $n>$ some $n_{0}(\omega)$ for almost all $\omega$.

Next, in order to compute the joint distribution of $\left(\tau_{n}^{(-)}, m_{n}\right)$, we exploit the fact that the event $\left\{\tau_{n}^{(-)}=k, m_{n}=s r^{m-1}\right\}$ occurs precisely when $k$ of the summands are equal to $s r^{m-1}$ and all the others are at least equal to $s r^{m}$, namely,

$$
\mathbb{P}\left(\tau_{n}^{(-)}=k, m_{n}=s r^{m-1}\right)=\left(\begin{array}{l}
n \\
k
\end{array}\right)\left(p q^{m-1}\right)^{k}\left(q^{m}\right)^{n-k}=\left(\begin{array}{l}
n \\
k
\end{array}\right) p^{k} q^{m n-k} .
$$

Summing over $m$ yields (as might be expected) a zero-truncated binomial distribution:

$$
\begin{aligned}
\mathbb{P}\left(\tau_{n}^{(-)}=k\right) & =\sum_{m=1}^{\infty}\left(\begin{array}{l}
n \\
k
\end{array}\right) p^{k} q^{m n-k} \\
& =\left(\begin{array}{l}
n \\
k
\end{array}\right)\left(\frac{p}{q}\right)^{k} \sum_{m=1}^{\infty} q^{m n} \\
& =\left(\begin{array}{l}
n \\
k
\end{array}\right) \frac{p^{k} q^{n-k}}{1-q^{n}}, \quad k=1, \ldots, n .
\end{aligned}
$$

By comparing the product of the marginal distributions of $\tau_{n}^{(-)}$and $m_{n}$ with (6.2), we see that the two quantities are independent. This is explained by the scale invariance of the St. Petersburg distribution, or otherwise put, the lack of memory of the geometric distribution. Moreover, by exploiting (6.3), we conclude first that $\mathbb{E}\left[\tau_{n}^{(-)}\right]=n p /\left(1-q^{n}\right)$ and $\operatorname{var}\left(\tau_{n}^{(-)}\right)=n p q /\left(1-q^{n}\right)$. From these moments, it follows that, as $n \rightarrow \infty$,

$$
\frac{\tau_{n}^{(-)}}{n} \stackrel{\text { a.s. }}{\rightarrow} p \quad \text { and } \quad \frac{\tau_{n}^{(-)}-n p}{\sqrt{n p q}} \stackrel{\mathrm{D}}{\rightarrow} N(0,1) .
$$

Finally, by combining (6.1) and the strong law for $\tau_{n}^{(-)}$, we immediately see that

$$
\left(\frac{\tau_{n}^{(-)}}{n}, m_{n}\right) \stackrel{\text { a.s. }}{\longrightarrow}(p, s) \quad \text { as } n \rightarrow \infty \text {. }
$$




\subsection{Asymptotics}

In this subsection we record asymptotic results for min-trimmed sums, trimmed sums, and the total minimum. To that end, set $N=r^{n}=q^{-n}$, and define $Z(t)$ as in Theorem 2.2. Coupled with the almost-sure convergence properties noted in the previous subsection we deduce analogues of Theorems 2.1 and 2.2 as follows.

Theorem 6.1. It holds that

$$
\frac{S_{t N}-m_{t N}^{*}}{N}-p s t n \stackrel{\mathrm{D}}{\rightarrow} Z(t)-p s t \quad \text { as } n \rightarrow \infty .
$$

Theorem 6.2. It holds that

$$
\frac{S_{t N}-m_{t N}}{N}-p s t n \stackrel{\mathrm{D}}{\rightarrow} Z(t) \quad \text { as } n \rightarrow \infty .
$$

Combining the exponential decay of $m_{n}$, namely, $\mathbb{P}\left(m_{n}>s\right)=q^{n}$, with (6.4), yields

$$
\frac{m_{n}^{*}}{n} \stackrel{\text { a.s. }}{\longrightarrow} p s \quad \text { and } \quad \frac{m_{n}^{*}-n p s}{\sqrt{n}} \stackrel{\mathrm{D}}{\rightarrow} N\left(0, s^{2} p q\right) \quad \text { as } n \rightarrow \infty .
$$

\section{Extreme trimming}

By combining our results for max- and min-trimming, the following mixtures can be obtained. They can be interpreted as maxmin analogues of intermediate trimming.

For $t>0$ and $n \rightarrow \infty$,

$$
\begin{aligned}
& \frac{S_{t N}-M_{t N}^{*}-m_{t N}^{*}}{N}-p s t n \stackrel{\mathrm{D}}{\rightarrow} Z^{*}(t)-t, \\
& \frac{S_{t N}-M_{t N}-m_{t N}^{*}}{N}-p s t n \stackrel{\mathrm{D}}{\rightarrow} Z_{*}(t)-t, \\
& \frac{S_{t N}-M_{t N}^{*}-m_{t N}}{N}-p \operatorname{stn} \stackrel{\mathrm{D}}{\rightarrow} Z^{*}(t), \\
& \frac{S_{t N}-M_{t N}-m_{t N}}{N}-p s t n \stackrel{\mathrm{D}}{\rightarrow} Z_{*}(t) .
\end{aligned}
$$

\subsection{The joint distribution of $\left(\tau_{n}^{(-)}, m_{n}, \tau_{n}^{(+)}, M_{n}\right)$}

The event $\left\{\tau_{n}^{(-)}=i, m_{n}=s r^{k-1}, \tau_{n}^{(+)}=j, M_{n}=s r^{m-1}\right\}$ occurs precisely when $i$ of the summands are equal to $s r^{k-1}, j$ of the summands are equal to $s r^{m-1}$, and all others lie strictly between $s r^{k-1}$ and $s r^{m-1}$. From this we deduce that

$$
\begin{aligned}
\mathbb{P}\left(\tau_{t N}^{(-)}\right. & \left.=i, m_{t N}=s r^{k-1}, \tau_{t N}^{(+)}=j, M_{t N}=s r^{m-1}\right) \\
& =\left(\begin{array}{l}
t N \\
i, j
\end{array}\right)\left(p q^{k-1}\right)^{i}\left(p q^{m-1}\right)^{j}\left(q^{k}-q^{m-1}\right)^{t N-i-j} .
\end{aligned}
$$

Since $m_{n} \stackrel{\text { a.s. }}{\longrightarrow} s$ as $n \rightarrow \infty$, the only nonzero contribution to the limit distribution comes from the case $k=1$. Noting also that $\left(\begin{array}{c}t N \\ i, j\end{array}\right)=\left(\begin{array}{c}t N \\ i\end{array}\right)\left(\begin{array}{c}t N-i \\ j\end{array}\right)$ yields

$$
\begin{aligned}
\mathbb{P}\left(\tau_{t N}^{(-)}=i, m_{t N}=s, \tau_{t N}^{(+)}=j, \frac{M_{t N}}{N}=s r^{m-1}\right) \\
\quad=\mathbb{P}\left(\tau_{t N}^{(-)}=i, m_{t N}=s, \tau_{t N}^{(+)}=j, M_{t N}=s r^{m+n-1}\right) \\
=\left(\begin{array}{c}
t N \\
i, j
\end{array}\right) p^{i}\left(p q^{m+n-1}\right)^{j}\left(q-q^{m+n-1}\right)^{t N-i-j}
\end{aligned}
$$




$$
\begin{aligned}
& =\left(\begin{array}{c}
t N \\
i
\end{array}\right)\left(\begin{array}{c}
t N-i \\
j
\end{array}\right) p^{i}\left(p q^{m+n-1}\right)^{j} q^{t N-i-j}\left(1-\frac{q^{m-2}}{N}\right)^{t N-i-j} \\
& =\left(\begin{array}{c}
t N \\
i
\end{array}\right) p^{i+j} q^{t N-i-j}\left(\begin{array}{c}
t N-i \\
j
\end{array}\right) q^{(n+m-1) j}\left(1-\frac{q^{m-2}}{N}\right)^{t N-i-j} .
\end{aligned}
$$

A consequence of the asymptotic normality of $\tau_{t N}^{(-)}$(cf. (6.4)) is that the contributing $i$-values reduce to $p t N \pm x \sqrt{p q t N}$. In the last displayed expression, the binomial probability from the first three factors converges to the probability mass of the corresponding normal distribution from (6.4), while, for the remaining factors,

$$
\begin{aligned}
\left(\begin{array}{c}
t N-i \\
j
\end{array}\right) q^{(n+m-1) j}\left(1-\frac{q^{m-2}}{N}\right)^{t N-i-j} & \\
& \sim\left(\begin{array}{c}
t N-t N p \\
j
\end{array}\right)\left(\frac{1}{N}\right)^{j} q^{(m-1) j}\left(1-\frac{q^{m-2}}{N}\right)^{t N-t N p-j} \\
& \sim\left(\begin{array}{c}
t N q \\
j
\end{array}\right)\left(\frac{1}{N q}\right)^{j} q^{m j}\left(1-\frac{q^{m-1}}{N q}\right)^{t N q-j} \\
& \sim \frac{(t N q)^{j}}{j !}\left(\frac{1}{N q}\right)^{j} q^{m j} \mathrm{e}^{-t q^{m-1}} \\
& =\frac{\left(t q^{m}\right)^{j}}{j !} \mathrm{e}^{-t q^{m-1}} .
\end{aligned}
$$

Using $\Phi$ to denote the standard normal distribution function, this shows that, as $n \rightarrow \infty$,

$$
\begin{gathered}
\mathbb{P}\left(\tau_{t N}^{(-)} \in(t N p+x \sqrt{t N p q}, t N p+y \sqrt{t N p q}], m_{t N}=s, \tau_{t N}^{(+)}=j, \frac{M_{t N}}{N}=s r^{m-1}\right) \\
\rightarrow \begin{cases}(\Phi(y)-\Phi(x)) \frac{\left(t q^{m}\right)^{j}}{j !} \mathrm{e}^{-t q^{m-1}} & \text { for }-\infty \leq x<y \leq \infty, j \in \mathbb{Z}_{+}, m \in \mathbb{Z}, \\
0 & \text { otherwise. }\end{cases}
\end{gathered}
$$

In particular,

- the pairs $\left(\tau_{t N}^{(-)}, m_{t N}\right)$ and $\left(\tau_{t N}^{(+)}, M_{t N}\right)$ are asymptotically independent;

- we rediscover the limit distribution of $\left(\tau_{t N}^{(+)}, M_{t N} / N\right)$ from Subsection 3.3; and

- $m_{t N}$ and $M_{t N}$ are asymptotically independent (which is no surprise, since $m_{t N} \stackrel{\text { a.s. }}{\longrightarrow} s$ as $n \rightarrow \infty)$.

\section{Almost-sure convergence?}

In this final section we offer some brief comments on almost-sure convergence. In view of (1.3) we can only expect a negative answer in the general case in the sense that (a version of) (1.3) remains true also in our framework. Indeed, for any $c>0$, we find, via (2.3), that $\sum_{n=1}^{\infty} \mathbb{P}\left(X_{n}>c n \log _{r} n\right)=\infty$ so that, by the Borel-Cantelli lemma, $\mathbb{P}\left(X_{n}>c n \log _{r} n\right.$ i.o. $)=1$ and, hence, all the more, $\mathbb{P}\left(S_{n}>c n \log _{r} n\right.$ i.o. $)=1$.

As for the limit inferior, following [1], we note, via partial integration and Lemma 2.1, that $\mu(x):=\int_{0}^{x} \mathbb{P}(X>y) \mathrm{d} y \sim s p \log _{r}(x / s)$ as $x \rightarrow \infty$, and, via (2.1), that $\mu(x) \sim \mu\left(x \log _{r} x\right)$ as $x \rightarrow \infty$. Finally, an application of [1, Theorem 2] tells us that $\liminf _{n \rightarrow \infty} S_{n} /\left(n \log _{r} n\right)=$ ps. 
Next, recalling [8], it is natural to ask whether almost-sure convergence holds for the normalized max-trimmed sum $S_{n}-M_{n}^{*}$. Unfortunately we have not been able to settle this problem beyond the triviality that

$$
0 \leq \liminf _{n \rightarrow \infty} \frac{S_{n}-M_{n}^{*}}{n \log _{r} n} \leq \limsup _{n \rightarrow \infty} \frac{S_{n}-M_{n}^{*}}{n \log _{r} n} \leq \limsup _{n \rightarrow \infty} \frac{S_{n}-M_{n}}{n \log _{r} n}=1 .
$$

However, by combining Theorems 3.1 and 3.2, we note that $M_{t N}^{*} / \log _{r} N \stackrel{\mathbb{P}}{\rightarrow} 0$ as $n \rightarrow \infty$; this implies that a Feller version holds, namely there is convergence in probability.

As for trimming from below, a Feller version for $S_{n}-m_{n}^{*}$ is immediate, in view of the strong law for $m_{n}^{*}$ in (6.5), but no almost-sure result holds.

\section{Acknowledgement}

We wish to thank an anonymous referee for a most thorough and careful reading of our manuscript.

\section{References}

[1] AdLer, A. (1990). Generalized one-sided laws of the iterated logarithm for random variables barely with or without finite mean. J. Theoret. Prob. 3, 587-597.

[2] Adler, A. And Rosalsky, A. (1989). On the Chow-Robbins "fair" games problem. Bull. Inst. Math. Acad. Sinica 17, 211-227.

[3] Berkes, I., Csáki, E. ANd Csörgő, S. (1999). Almost sure limit theorems for the St. Petersburg game. Statist. Prob. Lett. 45, 23-30.

[4] Berkes, I., Györfi, L. AND Kevei, P. (2017). Tail probabilities of St. Petersburg sums, trimmed sums, and their limit. J. Theoret. Prob. 30, 1104-1129.

[5] Chow, Y. S. and Robbins, H. (1961). On sums of independent random variables with infinite moments and "fair" games. Proc. Nat. Acad. Sci. USA 47, 330-335.

[6] Csörgő, S. (2002). Rates of merge in generalized St. Petersburg games. Acta Sci. Math. (Szeged) 68, 815-847.

[7] Csörgô, S. (2007). Merging asymptotic expansions in generalized St. Petersburg games. Acta Sci. Math. (Szeged) 73, 297-331.

[8] Csörgő, S. And Simons, G. (1996). A strong law of large numbers for trimmed sums, with applications to generalized St. Petersburg games. Statist. Prob. Lett. 26, 65-73.

[9] Feller, W. (1945). Note on the law of large numbers and "fair" games. Ann. Math. Statist. 16, 301-304.

[10] Feller, W. (1968). An Introduction to Probability Theory and Its Applications, Vol. I, 3rd edn. John Wiley, New York.

[11] Feller, W. (1971). An Introduction to Probability Theory and Its Applications, Vol. II, 2nd edn. John Wiley, New York.

[12] Gut, A. (2004). An extension of the Kolmogorov-Feller weak law of large numbers with an application to the St. Petersburg game. J. Theoret. Prob. 17, 769-779.

[13] Gut, A. (2010). Limit theorems for a generalized St. Petersburg game. J. Appl. Prob. 47, 752-760. (Correction: available at http://www.math.uu.se/ allan/86correction.pdf.)

[14] Gut, A. (2013). Probability: A Graduate Course, 2nd edn. Springer, New York.

[15] Gut, A. and Martin-Löf, A. (2015). Extreme-trimmed St. Petersburg games. Statist. Prob. Lett. 96, 341-345.

[16] Gut, A. and Martin-Löf, A. (2015). Generalized St. Petersburg games revisited. Preprint. Available at https://arxiv.org/abs/1506.09015v1.

[17] Gut, A. And Martin-Löf, A. (2016). A maxtrimmed St. Petersburg game. J. Theoret. Prob. 29, $277-291$.

[18] Martin-Löf, A. (1985). A limit theorem which clarifies the 'Petersburg paradox'. J. Appl. Prob. 22, 634-643.

\section{ALLAN GUT, Uppsala University}

Department of Mathematics, Uppsala University, Box 480, SE-751 06 Uppsala, Sweden.

Email address: allan.gut@math.uu.se

\section{ANDERS MARTIN-LÖF, Stockholm University}

Department of Mathematics, Stockholm University, SE-10691 Stockholm, Sweden.

Email address: andersml@math.su.se 